\title{
Mario Villa
}

L’applicabilité projective de deux transformations ponctuelles

Czechoslovak Mathematical Journal, Vol. 6 (1956), No. 4, 435-443

Persistent URL: http://dml.cz/dmlcz/100213

\section{Terms of use:}

(C) Institute of Mathematics AS CR, 1956

Institute of Mathematics of the Czech Academy of Sciences provides access to digitized documents strictly for personal use. Each copy of any part of this document must contain these Terms of use.

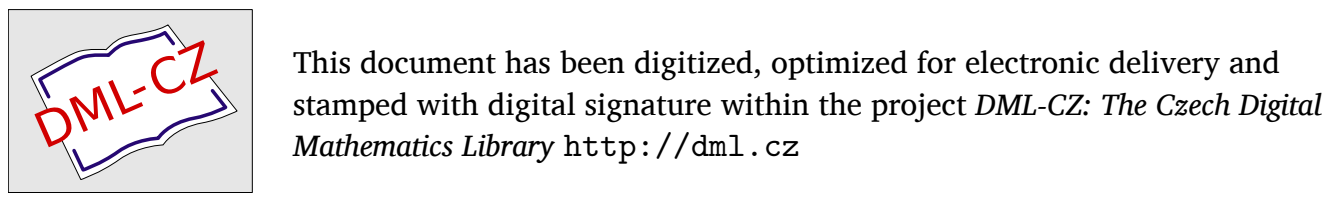




\title{
L'APPLICABILITÉ PROJECTIVE DE DEUX TRANSFORMATIONS PONCTUELLES
}

\author{
MARIO VILLA, Bologne.
}

Conférence faite le 3 septembre 1955 au IV ${ }^{\mathrm{e}}$ Congrès des mathématiciens tchécoslovaques à Prague.

1. La théorie projective différentielle des surfaces de l'espace ordinaire culmine avec la notion d'applicabilité projective et d'élément linéaire projectif.

Eh bien, comme nous allons montrer dans cette conférence, même dans la théorie des transformations ponctuelles entre deux plans, on peut introduire la notion d'applicabilité projective de deux transformations ponctuelles et d'élément linéaire projectif d'une transformation ponctuelle, qui joue dans cette théorie un rôle analogue à celui d'élément linéaire projectif des surfaces de l'espace ordinaire.

2. Je rappelle d'abord la notion d'homographie tangente à une transformation entre deux surfaces et de directions caractéristiques ou principales. Dans un espace linéaire projectif $S_{r}$, ayant $r$ dimensions, on donne une surface $\Sigma$. Nous indiquerons par $x$ l'ensemble des $r+1$ coordonnées homogènes $x_{0}, x_{1}, \ldots$, $x_{r}$ d'un point $P$ de $S_{r}$; la surface $\Sigma$ pourra alors être représentée analytiquement par les équations

$$
x=x(u, v),
$$

dans lesquelles $u, v$ sont deux paramètres et nous supposerons dans tout ce qui suit que les fonctions qui apparaissent aux seconds membres des (1) satisfont à toutes les conditions de régularité (dans les régions où elles sont définies) dont on aura besoin pour les considérations analytiques qui vont suivre.

Si l'on considère une seconde surface $\bar{\Sigma}$, dans un autre espace projectif à $r$ dimensions $S_{r}$, et si l'on donne une transformation (ou correspondance) ponctuelle $\gamma$ entre les surfaces $\Sigma, \bar{\Sigma}$, on pourra toujours représenter analytiquement la surface $\bar{\Sigma}$ au moyen des $y=y(u, v)$, (en indiquant par $y$ l'ensemble des $r+1$ coordonnées homogènes d'un point de $\overline{S_{r}} y_{0}, y_{1}, \ldots, y_{r}$ ) de sorte que deux points correspondants $P, \bar{P}$ dans $\gamma$, sur les surfaces $\Sigma, \bar{\Sigma}$, s'obtiennent pour les mêmes valeurs des paramètres $u, v$. 
On dira qu'une homographie $\Omega$ entre les deux espaces $S_{r} \bar{S}_{r}$, est tangente d'ordre $k$ (tangente tout court pour $k=1$ ) à la transformation $\gamma$ entre $\Sigma, \bar{\Sigma}$ au couple $P, \bar{P}$ de points correspondants, lorsque toute courbe $C$ tracée sur $\Sigma$ et passant par $P$ est changée par la transformation $\gamma$ et par $\Omega$ respectivement en deux courbes $\bar{C}$ et $\bar{C}^{\prime}$ qui ont en $\bar{P}$ un contact analytique d'ordre $k$ au moins, et en général d'ordre $k$ exactement. Il peut arriver que pour certaines courbes $C$, dont la tangente en $P$ a une direction particulière $t$, les courbes $\bar{C}$ et $\bar{C}^{\prime}$ aient en $\bar{P}$ un contact géométrique ou même analytique d'ordre plus grand que $k$, dans ce cas on dira respectivement que la direction $t$ tangente en $P$ à $C$ (et de même la direction $\bar{t}$ tangente en $\bar{P}$ à la courbe $\bar{C}$ ) est caractéristique ou bien principale en relation à la transformation $\gamma$ et a l'homographie $\Omega$ (tangente d'ordre $k) .{ }^{1}$ )

3. Considérons maintenant le cas où $r=3$ et les deux surfaces $\Sigma, \bar{\Sigma}$ ne sont pas des surfaces développables. On dit qu'une correspondance $\gamma$ est asymptotique lorsque elle change les courbes asymptotiques de $\Sigma$ en celles de $\bar{\Sigma}$. Cela posé, une applicabilité (ou déformation) projective entre deux surfaces $\Sigma, \bar{\Sigma}$, des espaces $S_{3}, \bar{S}_{3}$, c'est une correspondance asymptotique entre les deux surfaces $\Sigma, \bar{\Sigma}$ pour laquelle il existe, dans le couple générique $P, \bar{P}$ de points correspondants, au moins une homographie tangente pour laquelle les deux directions asymptotiques issues de $P$ (de $\bar{P}$ ) sont caractéristiques. ${ }^{2}$ )

On se propose maintenant d'introduire une notion d'applicabilité projective pour les transformations entre deux plans aussi. Mais il faut mettre plusieurs choses d'abord.

4. Je rappellerai que si $x, y$ sont les coordonnées cartésiennes (ou projectives) dans un plan $\Pi$ et $\bar{x}, \bar{y}$ les coordonnées analogues dans un autre plan $\bar{\Pi}$, alors la. variété des couples de points des deux plans, ou variété de Segre, est la variété $V_{4}$, ayant quatre dimensions, de l'espace linéaire $S_{8}$, dont les équations sont

$$
\begin{gathered}
X_{1}=x \bar{x}, X_{2}=x \bar{y}, X_{3}=y \bar{x}, X_{4}=y \bar{y} \\
X_{5}=x, X_{6}=y, X_{7}=\bar{x}, X_{8}=\bar{y}, X_{0}=1 .
\end{gathered}
$$

Je rappellerai aussi qu'il existe sur la variété de Segre deux systèmes de plans $\infty^{2}$. Ces plans représentent les couples que l'on obtient en fixant un point dans l'un des deux plans $\Pi, \bar{\Pi}$ et en l'associant à tous les points de l'autre plan. Une transformation ponctuelle $T$ entre les deux plans $\Pi, \bar{\Pi}$, dont les équations sont $\bar{x}=f(x, y), \bar{y}=\varphi(x y)$ est représentée sur $V_{4}$ par une surface dont les équations s'obtiennent en substituant dans les équations qui précèdent à $\bar{x}, \bar{y}, f, \varphi$ (cela est évident du reste, puisque une correspondance est une totalité $\infty^{2}$ de couples et l'on a done sur la $V_{4} \infty^{2}$ points qui forment justement une surface). Inversement, une surface de $V_{4}$ représente une transformation entre les deux plans.

1) L'on voie, par exemple: $E$. Čech, Géométrie projective différentielle des correspondances entre deux espaces, Čas. pro pěstování matematiky a fysiky, 74, 32-48 (1950).

2) G. Fubini et E. Čech, Geometria proiettiva differenziale, Bologna, Zanichelli Ed., 1926-27; G. Fubini et $E$. Cech, Introduction à la Géométrie différentielle des surfaces, Paris, Gauthier-Villars Ed., 1931. 
5. Je rappellerai encore que dans une transformation ponctuelle entre deux plans $\Pi, \bar{I}$, en un couple régulier de points correspondants (c'est-à-dire un couple à Jacobien $\neq 0$ ), il existe $\infty^{2}$ homographies tangentes et l'on a trois directions caractéristiques, qui sont les mêmes pour toutes les homographies tangentes.

Les directions caractéristiques peuvent aussi être définies comme les directions qui conservent les inflexions. Par chaque point $P$ de $\Pi$ on a trois directions caractéristiques distinctes issues de ce point (cela se produit en général; mais il peut arriver que ces directions soient coincidentes ou indéterminées). On appelle courbe caractéristique une courbe du plan $I$ (ou du plan $\bar{\Pi}$ ) telle que la tangente au point générique soit une des trois directions caractéristiques issues de ce point. ${ }^{3}$ ) Dans chacun des plans on a donc un 3-réseau de courbes caractéristiques.

La considération des 3-réseaux caractéristiques est fondamentale pour l'étude des transformations ponctuelles entre deux plans. ${ }^{4}$ )

6. On a vu qu'une transformation ponctuelle est représentée sur la $V_{4}$ de Segre par une surface $\Sigma$. Il est naturel de se demander: comment seront représentées sur $\Sigma$ les couples de courbes caractéristiques? C'est justement ce problème qui m'a conduit à une extension de la notion de courbes quasi-asymptotiques de Bompiani, aboutissant aux courbes quasi-asymptotiques à trois indices.

Considérons une variété $V_{k}(k>2)$ dans un espace $S_{r}$ et sur la variété $V_{k}$ une autre variété $V_{m}(1<m<k)$. On dira qu'une courbe $C$, tracée sur $V_{m}$, est quasi-asymptotique $\gamma_{p, q, s}$ lorsque au point générique de la courbe l'espace qui joint les trois espaces $S(p)$-osculateur à la $V_{k}, S(q)$-osculateur à la $V_{m}, S(s)$ osculateur à la courbe $C$, a une dimension qui est inférieure à l'ordinaire. ${ }^{5}$ )

Ayant posé cette notion, on a que: les couples de courbes caractéristiques de la transformation ponctuelle $T$ sont représentés sur la surface $\Sigma$ de la $V_{4}$ de Segre qui représente $T$ par les courbes quasi-asymptotiques à trois indices $\left.\gamma_{1,2,3} \cdot{ }^{6}\right)$

Sur une surface de la variété $V_{4}$ de Segre ${ }^{7}$ ) on a donc un système triple $\infty^{1}$ (un 3-réseau) de courbes quasi-asymptotiques $\gamma_{1,2,3}$ (il en passe trois par chaque

${ }^{3}$ ) On exclut dans ce travail le cas où, dans le couple générique des points correspondants, les directions caractéristiques soient coincidentes ou indéterminées.

4) L'importance de cette notion a été reconnue pour la première fois dans le travail: O. Boruivka, Sur les correspondances analytiques entre deux plans projectifs, Publ. de la Fac. des Sc. de l'Univ. Masaryk, n 72, Brno (1926).

$\left.{ }^{5}\right)$ M. Villa et $G$. Vaona, Varietà quasi-asintotiche a più indici e curve caratteristiche di una trasformazione puntuale, Atti Accad. Naz. Lincei Rend., (8), 8, 470—476 (1950).

$\left.{ }^{6}\right)$ M. Villa et G. Vaona, le travail de la note ${ }^{5}$ ).

7) Les surfaces de la $V_{4}$ de Segre qui représentent les transformations ponctuelles dégénérées et les surfaces de Veronese sont exclues. L'on voie: M. Villa, Sulle trasformazioni puntuali degeneri, Mem. Accad. Sci. Ist. Bologna, (9), 9, 19-26 (1941-42); M. Villa, Sulle direzioni caratteristiche di una trasformazione puntuale; Mem. Accad. Sci. Ist. Bologna, (10), 9, 7-19 (1943). 
point). Les trois directions tangentes dans un point $P$ de la surface aux trois $\gamma_{1,2,3}$ qui passent par $P$ seront dites directions quasi-asymptotiques. Une correspondance entre deux surfaces appartenant à $V_{4}$ de Segre sera dite quasiasymptotique lorsqu'elle change le 3-réseau de $\gamma_{1,2,3}$ de l'une dans celui de l'autre.

\%. Le problème de l'applicabilité projective de deux transformations $T_{1}, T_{2}$ entre deux couples de plans peut être posé en une forme beaucoup plus claire et intuitive, lorsqu'on représente les deux transformations sur la variété de Segre, puisqui'il se réduit alors au problème de l'applicabilité projective de deux surfaces appartenant à deux variétés de Segre. Les deux variétés de Segre pourraient aussi être supposées coïncidentes et l'on a ainsi le problème de l'applicabilité projective entre deux surfaces d'une variété de Segre.

J'ai rappelé comment interviennent d'une façon essentielle, dans le problème de l'applicabilité projective entre deux surfaces de l'espace ordinaire, les réseaux des courbes asymptotiques des deux surfaces. A présent, les 3-réseaux de courbes quasi-asymptotiques à trois indices $\gamma_{1,2,3}$ se substituent aux réseaux de courbes asymptotiques.

Avant de poser la notion d'applicabilité projective entre deux surfaces appartenant à deux variétés de Segre $V_{4}, V_{4}^{\prime}$, représentatives des couples de points de $\left(\Pi_{1}, \Pi_{2}\right),\left(\Pi_{1}^{\prime}, \Pi_{2}^{\prime}\right)$, il faut préciser que parmi les homographies qui changent l'une dans l'autre les deux variétés de Segre $V_{4}, V_{4}^{\prime}$ (ou bien la $V_{4}$ de Segre en elle-même) nous considérerons seulement celles qui changent un certain système de plans (parmi les deux qui existent sur la $V_{4}$ ) en un système de plans de $V_{4}^{\prime}$ déterminé. C'est-à-dire que nous considérons les $\infty^{16}$ homographies qui sont les images des couples d'homographies entre les plans $\Pi_{1}, \Pi_{1}^{\prime}$ et $\Pi_{2}, \Pi_{2}^{\prime}$. Nous indiquerons par $I$ l'ensemble des dites $\infty^{16}$ homographies qui changent l'une dans l'autre $V_{4}, V_{4}^{\prime}$.

Cela posé, nous donnons la définition suivante:

Une correspondance $\gamma$ entre deux surfaces $\Sigma, \Sigma^{\prime}$ situées sur des variétés de Segre, est une applicabilité projective lorsqu'elle est quasi-asymptotique et dans le couple générique $P, P^{\prime}$ de points correspondants et pour chacun des trois couples de directions quasi-asymptotiques il existe au moins une homographie tangente dans l'ensemble $I$ pour laquelle les deux directions dites sont caractéristiques. ${ }^{8}$ )

8. Evidemment il est souhaitable d'avoir une définition de l'applicabilité projective entre deux transformations ponctuelles pour laquelle on n'ait pas besoin de faire recours aux modèles sur la variété de Segre.

Soit donc $T$ une transformation ponctuelle entre les deux plans $\Pi_{1}, \Pi_{2}$ et $T^{\prime}$ une transformation ponctuelle entre les plans $\Pi_{1}^{\prime}, \Pi_{2}^{\prime}$. Une correspondance $\gamma$

$\left.{ }^{8}\right)$ Pour cette définition: $M$. Villa et $L$. Muracchini, L'applicabilità proiettiva di due trasformazioni puntuali (qui va paraitre dans le „Bollettino dell'Unione Matematica Italiana"). 
entre $T$ et $T^{\prime}$ consistera en une transformation $U_{1}$ entre les plans $\Pi_{1}, \Pi_{1}^{\prime}$ et une seconde transformation $U_{2}$ entre les plans $\Pi_{2}, \Pi_{2}^{\prime}$, cette dernière transformation étant induite par $T, T^{\prime}$ et $U_{1}\left(U_{2}=T . U_{1} . T^{\prime}\right)$. Eh bien, la définition que nous avons donnée de l'applicabilité projective peut être exprimée comme il suit: ${ }^{9}$ )

Etant données deux transformations, $T$ entre les plans $\Pi_{1}, \Pi_{2}$, et $T^{\prime}$ entre les plans $\Pi_{1}^{\prime}, \Pi_{2}^{\prime}$, une correspondance $\gamma$ entre $T$ et $T^{\prime}$ (consistant en une transformation $U_{1}$ entre $\Pi_{1}, \Pi_{1}^{\prime}$ et la transformation $U_{2}$ induite entre $\Pi_{2}, \Pi_{2}^{\prime}$ ) sera dite applicabilité projective si les 3-réseaux caractéristiques de $U_{1}, U_{2}$ dans les quatres plans sont les 3-réseaux caractéristiques de $T, T^{\prime}$.

Il s'ensuit que les 3-réseaux de $T, T^{\prime}$ dans les plans $\pi_{1}$, $\pi_{1}^{\prime}$, doivent être topologiquement équivalents.

9. Nous allons voir à présent quelle est la condition analytique pour l'applicabilité de deux transformations. Nous allons parvenir à la notion d'élément linéaire projectif d'une transformation qui comme l'on a déjà dit, joue dans la théorie des transformations un rôle semblable à celui de l'élément linéaire projectif dans la théorie projective différentielle des surfaces. Nous emploierons la méthode du repère mobile.

Une transformation ponctuelle $T$ entre deux plans $\Pi, \bar{\Pi}$ est déterminée lorsque on connaît les expressions des coordonnées homogènes du point $A$ de $\Pi$ et celles des coordonnées homogènes du point correspondant $B$ dans $\bar{\Pi}$ en fonctions (que nous supposerons analytiques) de deux paramètres $u_{1}, u_{2}$ (que nous appellerons principaux). Pour toutes les valeurs des $u_{1}, u_{2}$ que nous considérerons, nous supposerons que

$$
\left|A \frac{\partial A}{\partial u_{1}} \frac{\partial A}{\partial u_{2}}\right| \neq 0, \quad\left|B \frac{\partial B}{\partial u_{1}} \frac{\partial B}{\partial u_{2}}\right| \neq 0 .
$$

Dans le plan $\Pi$ prenons comme points fondamentaux du repère mobile le point $A$ et deux points arbitraires $A_{1}, A_{2}$. Dans le plan $\bar{\Pi}$ prenons comme points fondamentaux du repère mobile le point $B$ et les points correspondants des points $A_{1}, A_{2}$, qu'on indiquera par $B_{1}, B_{2}$, dans une homographie tangente fixée à l'avance $\Omega$.

On a les équation fondamentales

$$
\begin{array}{ll}
\mathrm{d} A=\omega_{00} A+\omega_{01} A_{1}+\omega_{02} A_{2}, & \mathrm{~d} B=\bar{\omega}_{00} B+\bar{\omega}_{01} B_{1}+\bar{\omega}_{02} B_{2}, \\
\mathrm{~d} A_{1}=\omega_{10} A+\omega_{11} A_{1}+\omega_{12} A_{2}, & \mathrm{~d} B_{1}=\bar{\omega}_{10} B+\bar{\omega}_{11} B_{1}+\bar{\omega}_{12} B_{2}, \\
\mathrm{~d} A_{2}=\omega_{20} A+\omega_{21} A_{1}+\omega_{22} A_{2}, & \mathrm{~d} B_{2}=\bar{\omega}_{20} B+\bar{\omega}_{21} B_{1}+\bar{\omega}_{22} B_{2},
\end{array}
$$

les $\omega_{i k}, \bar{\omega}_{i k}(i, k=0,1,2)$ étant des formes de Pfaff des deux paramètres principaux $u_{1}, u_{2}$ et des dix paramètres secondaires (trois coordonnées pour $A_{1}$, trois coordonnées pour $A_{2}$, deux pour l'homographie tangente et deux pour les

$\left.{ }^{9}\right)$ M. Villa et L. Muracchini, le travail de la note ${ }^{8}$ ). 
facteurs de proportionnalité des coordonnées de $A, B)$. Les équations de structure du groupe projectif sont

$$
\begin{aligned}
& {\left[\mathrm{d} \omega_{i k}\right]=\left[\omega_{i 0} \omega_{0 k}\right]+\left[\omega_{i 1} \omega_{1 k}\right]+\left[\omega_{i 2} \omega_{2 k}\right],} \\
& {\left[\mathrm{d} \bar{\omega}_{i k}\right]=\left[\bar{\omega}_{i 0} \bar{\omega}_{0 k}\right]+\left[\bar{\omega}_{i 1} \bar{\omega}_{1 k}\right]+\left[\bar{\omega}_{i 2} \bar{\omega}_{2 k}\right] .}
\end{aligned}
$$

$(0 \leqq i, k \leqq 2)$, en indiquant par $\left[\omega_{i j}, \omega_{j k}\right]$ le produit extérieur des deux formes $\omega_{i j}, \omega_{j k}$, et par $[\mathrm{d} \omega]$ la différentielle extérieure.

Entre les 18 formes de Pfaff $\left[\omega_{i j}, \bar{\omega}_{j k}\right]$ il doit donc exister six relations linéaires que l'on obtiendra en exprimant que les points $B_{1}, B_{2}$ sont les correspondants des points $A_{1}, A_{2}$ dans une homographie tangente.

On a

$$
\begin{gathered}
\Omega A=B, \quad \Omega \mathrm{d} A=\omega_{00} B+\omega_{01} B_{1}+\omega_{02} B_{2}= \\
=\mathrm{d} B+\left(\omega_{00}-\bar{\omega}_{00}\right) B+\left(\omega_{01}-\bar{\omega}_{01}\right) B_{1}+\left(\omega_{02}-\bar{\omega}_{02}\right) B_{2} .
\end{gathered}
$$

Pour que $\Omega$ soit une homographie tangente, il faut que le point $\Omega \mathrm{d} A=\mathrm{d} B$ soit proportionnel à $B$, ce qui nous donne les deux relations linéaires

$$
\omega_{01}=\bar{\omega}_{01}, \quad \omega_{02}=\bar{\omega}_{02} .
$$

Les quatres relations linéaires restantes s'obtiennent par différentiation extérieure des deux précédentes. Posons $\omega_{01}=\omega_{1}, \omega_{02}=\omega_{2}$ et remarquons que $\omega_{1}, \omega_{2}$ sont deux combinaisons linéaires indépendantes des différentielles des deux paramètres principaux (puisque la position géométrique de $A$ est déterminée par ces deux paramètres principaux).

Posons aussi $\tau_{i k}=\bar{\omega}_{i k}-\omega_{i k}$. Cela posé, par différentiation extérieure des (2), en tenant compte des équations de structure, on obtient

$$
\left[\omega_{1} \tau_{11}-\tau_{00}\right]+\left[\omega_{2} \tau_{21}\right]=0 ; \quad\left[\omega_{1} \tau_{12}\right]+\left[\omega_{2} \tau_{22}-\tau_{00}\right]=0 .
$$

D'après un lemme classique de Cartan il suit de là qu'il existe deux formes quadrátiques des $\omega_{1}, \omega_{2}$

$$
\Omega_{1}=c_{11}^{1} \omega_{1}^{2}+2 c_{12}^{1} \omega_{1} \omega_{2}+c_{22}^{1} \omega_{2}^{2}, \quad \Omega_{2}=c_{11}^{2} \omega_{1}^{2}+2 c_{12}^{2} \omega_{1} \omega_{2}+c_{22}^{2} \omega_{2}^{2}
$$

telles que

$$
\begin{aligned}
& \tau_{11}-\tau_{00}=\frac{1}{2} \frac{\partial \Omega_{1}}{\partial \omega_{1}}, \quad \tau_{21}=\frac{1}{2} \frac{\partial \Omega_{1}}{\partial \omega_{2}} \\
& \tau_{12}=\frac{1}{2} \frac{\partial \Omega_{2}}{\partial \omega_{1}}, \quad \tau_{22}-\tau_{00}=\frac{1}{2} \frac{\partial \Omega_{2}}{\partial \omega_{2}} .
\end{aligned}
$$

Ces relations sont les quatre relations linéaires entre nos formes de Pfaff. Les formes $\Omega_{1}, \Omega_{2}$ ont une importance fondamentale dans la géométrie projective différentielle des transformations ponctuelles.

10. A côté des formes quadratiques $\Omega_{1}, \Omega_{2}$ nous sommes parvenus récemment à deux formes cubiques $\Theta_{1}, \Theta_{2}$ d'intérêt fondamental elles-aussi. 
Remarquons que les formes $\omega_{1}, \omega_{2}$ peuvent être considérées comme des coordonnées homogènes d'une droite du faisceau $A$, déterminée par les points $A$ et $\omega_{1} A+\omega_{2} A_{2}$, c'est-à-dire $A$ et $\mathrm{d} A$. Et de même les $\omega_{1}, \omega_{2}$ peuvent être considérées comme des coordonnées homogènes de la droite du faisceau $B$, déterminée par les points $B$ et $\omega_{1} B_{1}+\omega_{2} B_{2}$, c'est-à-dire par $B$ et $\mathrm{d} B$.

Cela posé, l'équation des directions caractéristiques est $\omega_{1} \Omega_{2}-\omega_{2} \Omega_{1}=0$. Nous allons maintenant particulariser ultérieurement les repères projectifs. Prenons les points $A_{1}, A_{2}\left(B_{1}, B_{2}\right)$ sur deux droites caractéristiques et comme homographie tangente $\Omega$ l'homographie caractéristique relative aux droites $A A_{1}, A A_{2} \cdot{ }^{10}$ )

On démontre que cela entraîne $c_{11}^{1}=c_{22}^{1}=c_{11}^{2}=c_{22}^{2}=0\left(c_{12}^{1} \neq 0, c_{12}^{2} \neq 0\right)$. Mais par une ultérieure particularisation des repères mobiles on peut supposer que $c_{12}^{1}=c_{12}^{2}=-1$.

Les (3) deviennent alors

$$
\begin{gathered}
\tau_{11}-\tau_{00}=-\omega_{2}, \quad \tau_{21}=-\omega_{1}, \\
\tau_{12}=-\omega_{2}, \quad \tau_{22}-\tau_{00}=-\omega_{1} .
\end{gathered}
$$

Cherchons les six nouvelles relations qui doivent lier les formes $\omega$. Par différentiation extérieure des (4) on obtient

$$
\begin{gathered}
{\left[\left(2 \omega_{12}-2 \tau_{10}-\omega_{2}\right) \omega_{1}\right]+\left[\left(\omega_{22}-\omega_{00}-\omega_{21}-\tau_{20}\right) \omega_{2}\right]=0,} \\
{\left[\left(\omega_{22}-\omega_{00}-\omega_{21}-\tau_{20}\right) \omega_{1}\right]+\left[\left(2 \omega_{21}-\omega_{1}\right) \omega_{2}\right]=0,} \\
{\left[\left(2 \omega_{12}-\omega_{2}\right) \omega_{1}\right]+\left[\left(\omega_{11}-\omega_{00}-\omega_{12}-\tau_{10}\right) \omega_{2}\right]=0,} \\
{\left[\left(\omega_{11}-\omega_{00}-\omega_{12}-\tau_{10}\right) \omega_{1}\right]+\left[\left(2 \omega_{21}-2 \tau_{20}-\omega_{1}\right) \omega_{2}\right]=0 .}
\end{gathered}
$$

D'après le lemme de Cartan déjà rappelé il suit qu'il existe deux formes cubiques

$$
\begin{aligned}
& \Theta_{1}=\alpha_{30} \omega_{1}^{3}+3 \alpha_{21} \omega_{1}^{2} \omega_{2}+3 \alpha_{12} \omega_{1} \omega_{2}^{2}+\alpha_{03} \omega_{2}^{3}, \\
& \Theta_{2}=\beta_{30} \omega_{1}^{3}+3 \beta_{21} \omega_{1}^{2}+3 \beta_{12} \omega_{1} \omega_{2}^{2}+\beta_{03} \omega_{2}^{3}
\end{aligned}
$$

telles que l'on ait

$$
\begin{gathered}
2 \omega_{12}-2 \tau_{10}-\omega_{2}=\frac{1}{6} \frac{\partial^{2} \Theta_{1}}{\partial \omega_{1}^{2}}, \quad \omega_{22}-\omega_{00}-\omega_{21}-\tau_{20}=\frac{1}{6} \frac{\partial^{2} \Theta_{1}}{\partial \omega_{1} \partial \omega_{2}} \\
2 \omega_{21}-\omega_{1}=\frac{1}{6} \frac{\partial^{2} \Theta_{1}}{\partial \omega_{2}^{2}}, \quad 2 \omega_{12}-\omega_{2}=\frac{1}{6} \frac{\partial^{2} \Theta_{2}}{\partial \omega_{1}^{2}}, \\
\omega_{11}-\omega_{00}-\omega_{12}-\tau_{10}=\frac{1}{6} \frac{\partial^{2} \Theta_{2}}{\partial \omega_{1} \partial \omega_{2}}, \quad 2 \omega_{21}-2 \tau_{20}-\omega_{1}=\frac{1}{6} \frac{\partial^{2} \Theta_{2}}{\partial \omega_{2}^{2}} .
\end{gathered}
$$

Ces relations sont les six relations que nous cherchions.

On peut choisir sur les deux droites caractéristiques $A A_{1}, A A_{2}$ les deux points $A_{1}, A_{2}$ d'une manière intrinsèque de sorte que l'on ait' $\left.{ }^{11}\right) \alpha_{30}=3 \beta_{21}, \beta_{03}=3 \alpha_{12}$.

10) M. Villa, Problemi integrali sulle trasformazioni puntuali, Compositio Math., 12, 137 -146 (1954).

11) $M$. Villa, le travail de la note ${ }^{10}$ ). 
Considérons la forme différentielle fractionnaire $\Phi=\frac{\omega_{1} \Theta_{2}-\omega_{2} \Theta_{1}}{\omega_{1} \Omega_{2}-\omega_{2} \Omega_{1}}$, que nous appellerons, pour des raisons évidentes d'analogie, l'élément linéaire projectif de la transformation.

On démontre alors que: ${ }^{12}$ )

La condition nécessaire et suffisante pour que deux transformations $T, T^{\prime}$ soient projectivement applicables est qu'elles aient le même elément linéaire projectif.

Il est clair que c'est là un résultant important dans la théorie des transformations ponctuelles. Il y aurait beaucoup de choses à dire sur cet élément linéaire projectif (par exemple sur son interprétation géométrique), sur les formes $\Theta_{1}, \Theta_{2}$ et sur l'extension des notions introduites aux transformations ponctuelles entre espaces de dimension $>2$, mais je m'arrêterai ici.

\section{Резюме}

\section{ПРОЕКТИВНАЯ НАЛОЖИМОСТЬ ТОЧЕЧНЫХ СООТВЕТСТВИЙ}

МАРИО ВИЛЛА (Mario Villa), Болоня.

Прочитано на IV съезде чехословацких математиков в Праге 3/IX 1955 г.

Как известно, проективную наложимость между двумя неразвертывающимися поверхностями пространства $S_{3}$ можно определить как такое соответствие, которое сохраняет асимптотические линии и которое обладает тем свойством, что для каждой пары соответствующих друг другу точек существует в $S_{3}$ коллинеация, которая является для данной пары точек касательной, а для асимптотических направлений - соприкасающейся. Систему всех $\infty^{4}$ пар $A, B$, где $A$ пробегает плоскость $S_{2}$, а $B$ плоскость $\bar{S}_{2}$, можно интерпретировать как четырехмерное многообразие $V_{4}$ (многообразие Сегре) пространства $S_{8}$. Соответствие $T$ между плоскостями $S_{2}$, $S_{2}^{\prime}$ можно принять за поверхность $\Sigma$, погруженную в многообразие Сегре $V_{4}$. Характеристическим кривым соответствия $T$ соответствуют квазиасимптотические кривые $\gamma_{123}$ на поверхности $\Sigma$, погруженной в $V_{4}$. Преобразованию $T \rightarrow \bar{T}$, которое переводит соответствие $T$ в аналогичное соответствие $T$, соответствует преобразование $\Sigma \rightarrow \bar{\Sigma}$, переводящее поверхность $\Sigma$ в аналогичную поверхность $\bar{\Sigma}$. Руководствуясь аналогичным положением в случае двух поверхностей в $S_{3}$, назовем преобразование $\Sigma \rightarrow \bar{\Sigma}$ проективной наложимостью, если оно сохраняет квазиасимптотические кривые $\gamma_{123}$ и если оно обладает свойством, что для каждой пары соответствующих друг другу точек существует в $S_{8}$ коллинеация, которая сохраняет многообразие Сегре $V_{4}$, является касательной для преобразо-

$\left.{ }^{12}\right)$ M. Villa et L. Muracchini, le travail de la note ${ }^{8}$ ). 
вания поверхности $\Sigma$ в поверхность $\bar{\Sigma}$, а соприкасающейся для квазиасимптотических направлений. Это понятие проективной наложимости $\Sigma \rightarrow \bar{\Sigma}$ можно перенести и на проективную наложимость $T \rightarrow T$, которую можно определить и непосредственно без перехода к поверхностям в многообразии Сегре $V_{4}$. Аналитическим условием для того, чтобы две поверхности в $S_{3}$ находились в проективной наложимости является, как известно, инвариантность проективного́ линейного элемента. Также и в рассматриваемом случае можно проективную наложимость характеризовать инвариантностью проективного линейного элемента, который опять имеет вид дроби, в читателе которой на этот раз стоит биквадратичная, а в знаменателе кубическая дифференциальная форма. 